\title{
调控方式影响跨期选择的神经基础：内侧前额叶的 中介作用
}

郭逸群 ${ }^{(1)}$, 冯廷勇 ${ }^{(1) * *}$

(1) 西南大学心理学与社会发展研究中心, 重庆 400715 ;

(2) 西南大学心理学部认知与人格教育部重点实验室, 重庆 400715

*联系人, E-mail: fengty0@swu.edu.cn

2016-06-27 收稿, 2016-09-05 修回, 2016-09-08 接受, 2016-09-29 网络版发表 重庆市人文社会科学重点研究基地重点项目(16SKB007)、中央高校基本科研业务费专项(SWU1609321)、国家自然科学基金(31271117, 31571128)和中央高校基本科研业务经费创新团队项目(SWU1509392)资助

摘要跨期选择(intertemporal choice)是指个体对发生在不同时间点的成本与收益进行权衡, 进而做出的各种判 断和选择. 先前的研究表明, 个体在长久生活中所形成的调控方式(“评估”和“行动”定向)会对跨期选择产生影响, 然而调控方式影响跨期选择的认知神经机制依然不清楚. 为此, 本研究试图采用功能性磁共振成像(fMRI)技术考 察调控方式影响跨期选择的神经基础. 行为结果发现，“评估”定向者比“行动”定向者表现出更小的延迟折扣率; 被试的“评估”定向得分和延迟折扣率成负相关，而“行动”定向得分和延迟折扣率呈正相关. 脑成像的结果发现， 表征延迟奖常主观价值的内侧前额叶的激活水平与“评估”定向得分成显著的正相关，而与“行动”定向得分存在显 著的负相关; 进一步的中介分析表明，表征主观价值的内侧前额叶在调控方式与延迟折扣之间存在显著的部分中 介效应。这些结果说明，调控方式对跨期选择的影响可能是通过改变延迟奖赏的主观价值来实现的，而表征主观 价值的内侧前额叶则是其神经基础.

关键词跨期选择, 调控方式, 评估定向, 行动定向, 内侧前额叶, 功能性磁共振成像(fMRI)

跨期选择(intertemporal choice)是指个体对发生 在不同时间点的成本与收益进行权衡, 进而做出的 各种判断和选择 ${ }^{[1]}$. 亚当.斯密指出跨期选择不仅影 响一个人的健康、财富与整体幸福感, 也决定一个国 家的经济繁荣的程度 ${ }^{[1]}$. 从个人健康、金融投资, 到 国家的可持续发展、全球气候变暖等都包含着很强的 跨期因素. 广泛应用于消费、储蓄、投资、经济增长 等领域的理论建模与分析, 对于时间管理、薪酬管 理、各类成瘾行为 (吸烟、吸毒以及奢博)和暴饮暴食 症等精神疾病的治疗都具有实际的指导意义 ${ }^{[2,3]}$. 跨 期选择研究的一个重要发现是, 与当前或近期的获
益(或损失)相比, 人们总是倾向于赋予将来获益(或 损失)更小的权重, 这一现象叫做延迟折扣(delay discounting). 延迟折扣是跨期选择的核心指标，它 表征了个体对于及时奖赏或延迟奖赏的偏好程度： 延迟折扣率越高表示个体越倾向于选择及时奖赏, 而延迟折扣率越小则表示越倾向于选择延迟奖赏. 先前的研究表明，跨期选择会受到一些特质性因素 的影响，例如自我控制能力、时间洞察力、预期想象 能力等 ${ }^{[49]}$. 同时, 已有研究表明, 自我调控方式作 为一种重要的人格特质也会对跨期选择产生影 响 ${ }^{[10,11]}$. 
日常生活中, 有些人做出决策时, 会对各种方案 进行一一比较, 之后选择最好的一个才开始行动; 而 有些人正好相反, 做事情雷厉风行, 行动之前较少去 衡量各种方案的优缺点, 这就是两种不同自我调控 方式个体的典型表现. 所谓自我调控 (self-regulation), 是指个体明确自己的现实状态和目标状态, 以 及从现实状态到达目标状态的方法和手段, 然后付 诸实际行动. 从这个概念可以看出自我调控的两个 基本功能: 一是评估自己想要达到的目标状态以及达 到目标状态的手段和方法; 二是个体朝向目标努力. 在人们追求目标的过程中, 几乎都包含“评估”和“行 动”这两种最基本的调控方式定向。“评估”定向 (assessment orientation)指的是自我调控中比较的方 面, 主要关注于对各种目标和方法进行比较, 衡量各 种备选方案的优缺点, 然后做出最好的选择 ${ }^{[12]}$; 相 反, “行动”定向(locomotion orientation) 指的是状态之 间的转换, 关注的是怎样尽快地参与到活动中去, 在 这一过程中很少出现分心和延迟 ${ }^{[12]}$. 调控方式理论 (regulatory mode theory)认为“评估”和“行动”这两个 维度是相互独立的, 并且在不同的人身上表现出稳 定的个体差异 ${ }^{[12 \sim 14]}$.

调控方式作为一种重要的人格特质会影响到人 们的多种行为, 比如跨期决策、风险决策、反事实思 维等 ${ }^{[10,11,15 ~ 17]}$. 先前的研究表明, 高“评估”定向者 (评估者)为了做出更优的决策, 会遵循严格的评价准 则, 对各个选项进行一一比较 ${ }^{[13]}$. 研究者认为, 这种 特质会使评估者可能更加重视长远的利益, 也表现 为更加 “目光远大”. 而高 “行动”定向者(行动者)在面 临选择时会很快的做出决策, 以便尽早参与到行动 中, 对他们来说等待是不可取的, 因此在做决策时可 能缺少长远的考虑, 显得“目光短浅, ”[18]. 已有研究从 行为上证实: 在面对跨期选择时, 评估者会更多的选 择延迟大奖赏, 表现出更小的延迟折扣率; 而行动者 则会更多的选择立即小奖赏, 表现出更大的延迟折 扣率 ${ }^{[10,11]}$. 另外, 研究人员使用静息态功能连接的技 术发现, 外侧前额叶 (lateral prefrontal cortex, IPFC)与 内侧前额叶 (medial prefrontal cortex, mPFC)的功能 连接在调控方式定向与延迟折扣率之间起着显著的 中介作用 ${ }^{[11]}$. 但是静息态功能连接的技术并不能深 人探讨个体在完成具体任务时大脑的活动模式. 因 此, 调控方式影响跨期选择的认知神经机制依然不 清楚.
先前的研究表明在面临决策时, 评估者会优先 关注选项的价值而不是选项的可得性，而行动者却 会优先关注选项可得性而不是价值 ${ }^{[12,14]}$. 这些研究 表明, 评估者对决策的价值可能更加敏感, 表征价值 的网络的神经活动可能更强; 而行动者对决策价值 的敏感性不高, 相应的表征价值的网络的神经活动 可能更弱. 而关于跨期选择的神经机制, Kable 等 人 ${ }^{[19,20]}$ 认为, 大脑中存在一个以双曲线折扣形式编 码奖赏的主观价值系统(价值表征网络), 个体通过比 较主观价值表征的强弱来决定选择立即奖赏还是延 迟奖赏的; 价值表征网络包括内侧前额叶(mPFC)、腹 侧纹状体 (ventral striatum, VS) 和后扣带皮层 (posterior cingulate cortex, PCC)等脑区 ${ }^{[21 ~ 23]}$. 价值表 征网络是从价值加工的过程来解释跨期选择的, 并 且这些脑区的活动可以预测个体的延迟折扣率 ${ }^{[19]}$. 后来的研究者通过操纵不同的奖赏数量、不同的研究 范式、不同的刺激类型都发现这些脑区表征的是奖赏 的主观价值 ${ }^{[6,22,23]}$. 这些证据进一步支持跨期选择中 存在一个主观价值表征的网络. 因此, 本研究着重探 讨跨期选择中的价值表征网络在调控方式影响跨期 决策中的作用. 由于评估者决策时更加偏爱选项的 价值, 本研究预期他们在跨期选择中所表现出的较 小的延迟折扣率可能是由于他们的价值表征网络(或 价值表征网络中的某个脑区)的活动更强造成的; 而 行动者决策时较少关注选项的价值, 本研究预期他 们在跨期选择中所表现出的较大的延迟折扣率可能 是由于他们的价值表征网络(或价值表征网络中的主 要脑区)的活动较弱造成的.

本研究试图采用功能性磁共振成像技术(fMRI), 考察调控方式影响跨期选择的神经机制, 尤其是价 值表征网络的作用. 首先, 采集被试完成跨期选择任 务的脑成像数据. 其次, 根据双曲线折扣算出延迟奖 赏的主观价值, 再使用参数调节 (parametric modulation）的分析方法定位表征主观价值的脑区；最后， 使用中介分析的方法考察价值表征网络的活动是否 在调控方式影响跨期决策中起中介作用.

\section{1 材料与方法}

\section{1 被试}

共 86 名国内某大学本科生或研究生参加实验, 其中男生 44 人, 女生 42 人, 年龄范围为 18 26岁, 平 
均年龄 20.35 岁, 标准差为 1.87 岁. 排除了在任务态扫 描中头动大于 $3 \mathrm{~mm}$ 或 3 度的被试 3 人, 同时排除了全 部选择立即或延迟奖赏的被试 2 人, 最终剩余 81 人. 被试均为右利手, 没有生理或心理疾病, 自愿参加, 实验前均签署了实验知情同意书. 为了让被试更认 真地参与实验, 实验报酬是根据被试的真实选择进 行的. 实验结束后, 让被试随机抽取一个试次, 根据 被试在该试次上的选择给予报酬. 如果被试抽中试 次的选择是立即奖赏, 则立即支付与奖赏同等数量 的报酬; 如果抽中试次的选择是延迟奖赏, 则被试需 要等待相应的延迟时间才能得到与延迟奖常数量相 等的报酬.

\section{2 调控方式量表}

调控方式量表由Kruglanski等人 ${ }^{[12]}$ 编制, 共有 24 个题项: “评估”和“行动” 维度各 12 题(如“我花费大量 时间来反省我个性中存在的优点和缺点”), 采用六点 计分. 先前的研究表明, 该量表能够较好地测量个体 在评估定向和行动定向上的稳定差异, 具有较好的 信、效度 ${ }^{[24,25]}$.

\section{3 跨期选择任务}

跨期选择采用经典的跨期选择任务 ${ }^{[26]}$. 立即奖 赏为 10 29Y (平均为 $20 Y$ 的高斯分布), 延迟奖赏是 根据立即奖赏的增加比例 $(10 \%, 15 \%, 20 \%, 25 \%$, $35 \%, 50 \%, 70 \%, 95 \%, 125 \%)$ 来计算的. 共有3种延迟 时间: 一周、半个月、一个月. 被试共完成 72 个试次, 分两个run进行, 在被试间平衡 2 个run的先后顺序. 跨期选择任务采用E-prime 2.0编程, 电脑屏幕的左边 呈现立即奖赏, 右边呈现延迟奖赏. 实验流程见图 1 .

先前的研究表明, 曲线下面积 (area under the curve, AUC) 能够很好地反映被试的延迟折扣 ${ }^{[27 ~ 29]}$, 因此本研究采用AUC作为延迟折扣的指标. AUC 是 不基于任何模型的一个延迟折扣指标, 它能更加直 接地反映出个体对延迟奖赏的选择偏好, AUC越大 越偏爱延迟奖赏, 折扣率也就越小. 在计算 $\mathrm{AUC}$ 时, 首先算出每个延迟时间上的无差别点 (选择延迟奖赏 的概率为 0.5 时的延迟奖赏的数量即为无差别点, 使 用Logistic 回归拟合被试的选择, 然后求出在 0.5 时奖 赏数量), 将立即奖赏数量除以无差别点的值作为 discounting value(DV). 然后将延迟时间除以最大的 延迟时间作为延迟参数. 最后 $x$ 轴为延迟参数, $y$ 轴为

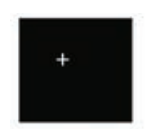

$1 \sim 5 \mathrm{~s}$

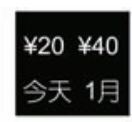

$4 \mathrm{~s}$

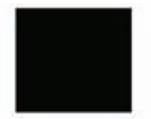

$1 \sim 5 \mathrm{~s}$

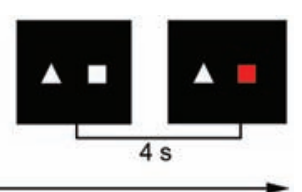

实验流程图
图 1 跨期选择任务实验流程图. 在每个试次中, 首先在屏幕中央呈 现“+”, 持续 1 5 s (jitter); 紧接着出现两种奖赏, 持续 $4 \mathrm{~s}$; 奖赏之后 出现一个持续 1 5 s (jitter)的空屏; 然后出现选择界面(三角和方形分 别代表立即奖赏和延迟奖赏, 两种条件在被试间平衡); 最后是反馈 界面(红色标记的为被试的选择), 选择和反馈阶段共 $4 \mathrm{~s}$.

Figure 1 Flow diagram of intertemporal choice task. Flow diagram of intertemporal choice task. On each trial, a fixation, which lasted 1-5 s, was first presented on the center of screen; two rewards were next appeared and lasted $4 \mathrm{~s}$; a blank screen lasting 1-5 s (jitter) was presented after the presentation of rewards; then participants were asked to make a choice between two rewards (triangle and rectangle were respectively represented immediate reward and delayed reward, and this was balanced between participants); feedback phase was finally showed (the red graph was indicated the participants' choice); choice and feedback phase were lasting for $4 \mathrm{~s}$

DV，将每个时间点上的DV值依次连接成梯形，梯形 面积之和即为 AUC, AUC的值在0 1之间. 同时, 由 于在计算延迟奖赏主观价值时需要用到折扣率 $k$, 在 本研究也采用双曲线公式 $S V=A /(1+k d)$ 拟合了延迟折 扣率 $k(S V$ 为折扣后的主观价值, $A$ 为客观的延迟奖赏, $d$ 为延迟时间). 双曲线折扣 $k$ 同样表征的是对不同奖 赏的选择偏好, $k$ 值越大，越偏好立即奖赏。在计算 $k$ 值时, 同样先计算出 $D V$ 值, 因为在无差别点时, 无 差别点的主观价值与立即奖赏数量相等, 所以公式 $S V=A /(1+k d)$, 可变为 $S V / A=1 /(1+k d)$, 而 $S V / A$ 即为 $D V$ 值, 也即 $D V=1 /(1+k d)$. 最后根据 $D V=1 /(1+k d)$ 拟合出 一个最佳的 $k$ 值. 将 $k$ 取对数后, 相关分析的结果显示 AUC 和 $\operatorname{logk}$ 存在很强的负相关 $(r=-0.955 ; P=0.000)$, 表明AUC对延迟折扣率具有良好的表征.

\section{4 fMRI数据收集与分析}

(i) 数据收集. 本研究中的脑成像数据是在一 台Siemens Trio 3T的磁共振仪器上收集的. 功能像的 扫描序列为 EPI(echo-planar imaging), 扫描参数: echo time $=30 \mathrm{~ms}$; repetition time $=2 \mathrm{~s}$; field of view $=$ $200 \mathrm{~mm} \times 200 \mathrm{~mm}$, matrix $=64 \times 64$; voxel size $=3.1 \times$ $3.1 \times 3$; 扫描层数为 33 ; 层厚为 $3 \mathrm{~mm}$. 对应的 $3 \mathrm{D}$ 结构 像的扫描序列为梯度回波脉冲序列(DPGR), 扫描参 数: repetition time $=2530 \mathrm{~ms}$; echo time $=3.39 \mathrm{~ms}$; flip angle $=9^{\circ}$; field of view $=256 \mathrm{~mm} \times 256 \mathrm{~mm}$; voxel size $=$ $1.3 \times 1.0 \times 1.3 \mathrm{~mm}^{3}$; 层数为 128 , 层厚为 $1.3 \mathrm{~mm}$.

(ii) 预处理. fMRI数据预处理使用的软件为 
$\mathrm{DPARSF}^{[30]}$, 该软件基于REST ${ }^{[31]}$ 和SPM8 (Wellcome Trust Centre for Neuroimaging, London, UK). 为了排 除刚开始扫描时所造成的被试的不适应, 我们剔除 了前三个时间点的数据, 以使数据更加稳定. 功能像 数据预处理包括时间校正, 头动校正 (以第一个 volume为基准), 再将头动校正后的图像与其相对应 的高分辨率的结构像进行配准, 其次将结构像分割 成灰质、白质和脑脊液, 然后根据分割的信息将功能 像做空间标准化(MNI空间). 为了进一步排除核磁扫 描时所产生的飘移信号, 进行了去线形漂移. 最后对 空间标准化后的数据进行平滑 (FWHM=8 mm). 将平 滑后的数据用于接下来的分析. 接下来的数据分析 使用的软件均为 SPM8(Wellcome Trust Centre for Neuroimaging, London, UK).

(iii) 参数调节分析. 参数调节分析是在为了定 位表征主观价值的脑区, 我们进行了参数调节分析 (parametric modulation). 首先根据双曲线折扣函数计 算了每个延迟奖赏的主观价值(subjective value, SV; $S V=A /(1+k d)$ ). 在建立 GLM (general linear model)模 型时, 我们将每一个阶段作为一个条件纳人到模型 中, 共 5 个, 并且在奖赏呈现阶段加人双曲线公式计 算出的每个延迟奖赏的主观价值 $(S V)$, 并把其作为主 观价值的调节参数; 又将 6 个方向的头动参数纳人到 模型中, 以进一步排除头动的影响. 建立模型后, 对 每个事件进行参数估计. 在组分析中, 使用单样本 $T$ 检验定位出表征主观价值的脑区, 取 $P<0.05$, FWE全 脑矫正. 最后, 为了进一步考察表征主观价值的脑区 是否是调控方式影响跨期选择的神经基础, 使用 ROIs (regions of interest)分析的方法进行验证. 将参 数分析中激活的内侧前额叶 (peak MNI coordinate: 0 39 -3)、腹侧纹状体 (peak MNI coordinate: -9 120 )、

丘脑 (peak MNI coordinate: $-15-249$ )和后扣带回 (peak MNI coordinate: $-3-33$ 30)这些脑区的团块作 为 ROIs. 在接下来的ROIs分析中, 先提取出了 ROIs 表征主观价值的Beta值, 然后将其作为中介变量, 以 检验表征主观价值的脑区是否在调控方式和跨期选 择之间起着中介作用.

(iv) 中介分析. 为了进一步检验调控方式影响 跨期选择的神经基础, 作者假设感兴趣区的神经活 动可能会在调控方式与跨期选择之间起着中介的作 用. 因此, 将感兴趣区的神经活动作为中介变量, 进 行中介分析. 中介分析使用SPSS中的间接效应检

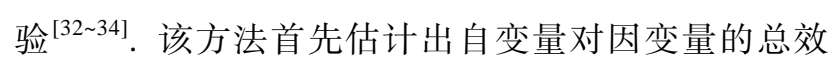
应、直接效应和间接效应. 然后使用bootstrap的方法 对间接效应进行检验，给出一个置信区间，如果该置 信区间包含 0 , 则间接效应不显著, 说明自变量不是 通过中介变量来影响因变量的; 而如果该置信区间 不包含 0 , 则间接效应显著, 说明自变量是通过或部 分通过中介变量来影响因变量的. 如果排除中介变 量的间接效应后，自变量对因变量的效应依然显著， 则为部分中介; 而如果排除间接效应后，自变量对因 变量的效应不显著, 则为完全中介效应.

(V) 验证性分析. 上述分析的结果发现, 表征 主观价值的内侧前额叶的活动在调控方式与跨期选 择之间起着部分的中介作用. 但是以上的数据分析, 根据组分析结果定ROI, 再进行ROI分析, 可能会产 生“依赖性”的问题 ${ }^{[35,36]}$, 从而高估了单个被试的效 应值. 为了排除“依赖性”, 使结果更加可靠, 本研究 进行了验证性分析. 由于本研究的样本量较大(共 81 人), 在验证性分析中使用了分半的方法, 将前一半 被试(40人; 男 29 , 女 11 ; 年龄为 17 24岁, 平均为 22.2 岁)用于定位分析, 将后一半被试(41人; 男 12 , 女 29 ; 年龄为 18 25岁, 平均为 20.5 岁)用于ROI分析. 在定位分析中, 同样建上述参数分析的 GLM, 定位 出表征主观价值的脑区. 在ROI分析中, 通过提取定 位分析中激活的脑区的Beta值, 将其作为中介变量, 进行中介分析, 以验证表征主观价值的脑区的活动 是否为调控方式影响跨期选择的神经基础.

\section{2 实验结果}

\section{1 行为结果}

首先, 为了排除两种调控方式定向可能对跨期 选择产生的交互影响，使用相关的方法考察了“评 估”定向和“行动”定向之间的关系. 结果表明, “评估” 得分和 “行动”得分之间的相关不显著 $(r=-0.010$; $P=0.927)$, 表明二者相互独立. 其次, 为排除调控方 式与跨期选择之间可能存在的混淆变量(例如性别、 年龄), 本研究考察了“评估”得分、“行动”得分、AUC 与年龄、性别之间的关系. 结果表明, 年龄与“评估” 得分、“行动”得分以及AUC的相关都不显著 $(P>0.05)$; “评估”得分、“行动”得分以及AUC这3个变量分别在 性别上的差异也均不显著 $(P>0.05)$. 这些结果说明, 年龄和性别因素不会影响到调控方式与跨期选择之 
间的关系.

为了考察两种调控方式定向对跨期选择产生的 影响, 本研究采用两种不同的方法来分析调控方式 与跨期选择之间的关系 ${ }^{[4,11]}$. 第一种方法将被试的两 种调控方式定向作为连续变量, 分别与 AUC进行相 关分析. 结果表明, “评估”得分和AUC存在显著的正 相关 $(r=0.304 ; P=0.006$. 如图 2(a)); 而 “行动”得分和 AUC存在显著的负相关 $(r=-0.332 ; P=0.002$. 如图 2(b)). 为进一步检验调控方式定向对跨期选择的影 响, 使用回归分析的方法, 将折扣率指标AUC作为 因变量, 将“评估”定向得分和“行动”定向得分作为自 变量同时进人回归方程中. 回归分析结果发现, 评估 定向得分和行动定向得分均能显著的预测折扣率(评 估定向: $b=0.301, t(78)=2.971, P=0.004$; 行动定向: $b=-0.329, t(78)=3.148, P=0.002)$. 第二种方法采用分 类比较的方法, 将被试按照其在“评估”和“行动”得分 上的相对高低, 先将“评估”和“行动”得分转化成 $Z$ 分 数, 把“评估”得分高于 “行动”得分的被试作为评估者 (43人), 反之, 把“行动”得分高于“评估”得分的被试 作为行动者 ( 38 人). 双样本 $T$ 检验的结果表明, 评估 者的 AUC 显著的高于行动者 $(t=2.990, d f=79, P=$ 0.004. 如图2(c)). 行为结果表明, “评估”定向越强的 个体, 其AUC值越大, 折扣率越小; 而“行动”定向越 强的个体, AUC值越小, 折扣率越大.

\section{2 脑成像结果}

为了定位表征主观价值的脑区, 将延迟奖赏的 主观价值作为一个参数, 进行参数调节分析. 结果发 现, 内侧前额叶 (mPFC), 腹侧纹状体 (VS), 丘脑
(thalamus)和后扣带皮层(PCC)存在显著的正激活(图 3及表1). 这些结果表明这些脑区在跨期选择中表征 了延迟奖赏的主观价值, 并且表明个体对延迟奖赏 的主观价值表征越大, 这些脑区的活动就越强 ${ }^{[19,20]}$. 为进一步考察调控方式定向与主观价值表征网络之 间的关系，进行了ROI分析. 在ROI分析时，由于丘 脑和纹状体是在同一个cluster中，所以我们选取了一 篇做主观价值表征元分析的腹侧纹状体的 mask ${ }^{[37]}$ 和 AAL模板中丘脑的 mask, 分别用这两个 mask与该 cluster取交集，从而得到纹状体和丘脑的ROI. 分别 提取以上激活脑区的Beta值，其表示的是这些脑区 表征主观价值的强弱，接下来将其与两种调控方式 定向分别进行相关分析. 结果发现: 表征主观价值的 内侧前额叶的激活水平与 “评估”得分呈显著的正相 关 $(r=0.322 ; P=0.003$; Bonferroni校正, 取 $P$ 小于 $0.05 / 4$ 为显著性水平, 下同), 而与 “行动”定向得分存在显 著的负相关 $(r=-0.294 ; P=0.008)$. 此外, 表征主观价 值的其他脑区活动与 “评估”得分或 “行动”得分的相 关均未达到显著水平(表2). 以上结果表明, 调控方 式定向与跨期选择中主观价值加工网络之间存在紧 密的联系, 且主要表现在表征主观价值的内侧前额 叶的活动上.

为进一步验证主观价值表征是否是调控方式影 响跨期选择的神经基础, 本研究进行了中介分析. 中 介分析使用SPSS中的间接效应检验，该方法需要估 计出总效应、直接效应和间接效应 ${ }^{[32 \sim 34]}$. 在中介分析 时，把表征主观价值的内侧前额叶的活动作为中介 变量, 以考察调控方式对跨期选择的影响是否通过 该中介变量实现的. 通过对间接效应的检验发现，在
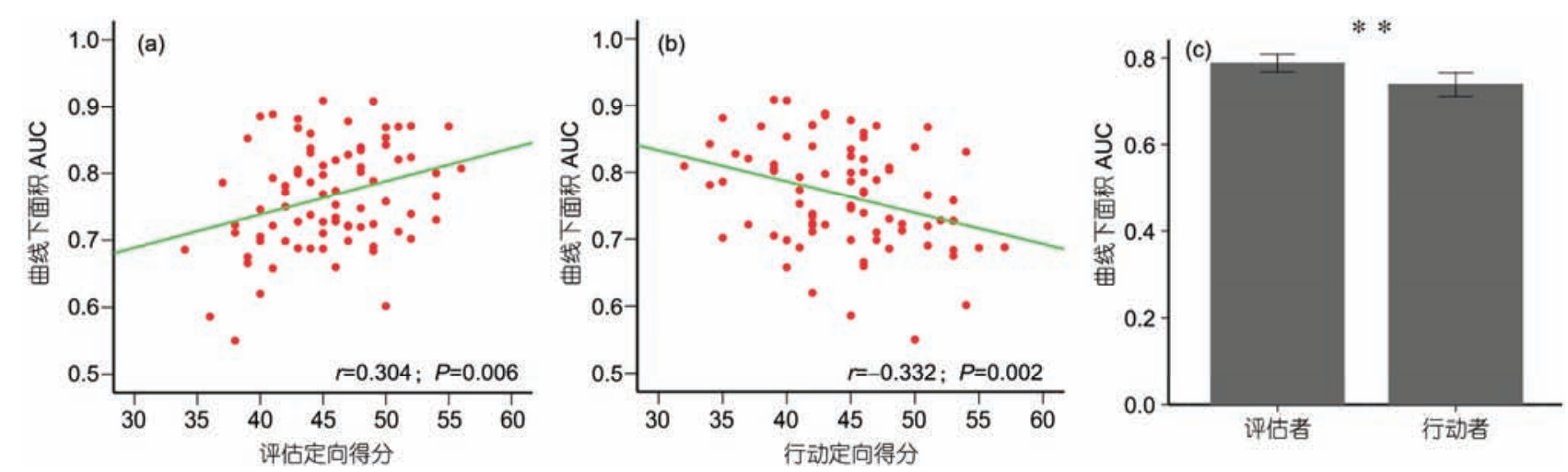

图 2 行为结果. (a) 为评估定向与 $\mathrm{AUC}$ 的相关图; (b) 行动定向得分与 $\mathrm{AUC}$ 的相关图; (c) 为评估者和行动者在 $\mathrm{AUC}$ 上的差异图 $(* *, P<0.01)$ Figure 2 Behavioral results. (a) Positive correlation between AUC and assessment orientation; (b) negative correlation between AUC and locomotion orientation; (c) locomotors showed a significantly lower AUC than assessors (**, $P<0.01)$ 


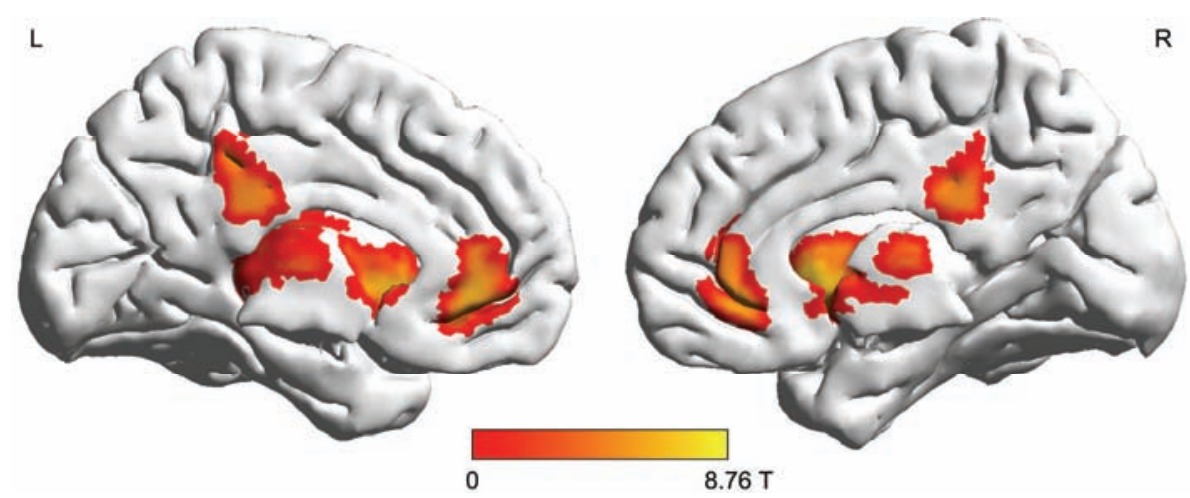

图 3 表征延迟奖赏主观价值的激活脑区 $(P<0.05$, FWE全脑矫正, cluster size $>10)$

Figure 3 Brain regions that significantly represented the subjective value of delayed reward $(P<0.05$, FEW corrected, cluster size $>10)$

表 1 主观价值表征的激活脑区 ${ }^{\mathrm{a}}$

Table 1 Activated region that represented the subjective value

\begin{tabular}{|c|c|c|c|c|c|c|}
\hline 脑区 & 布鲁德曼分区 & 体素个数 & 激活最强点 $T$ 值 & $x$ & $y$ & $z$ \\
\hline 双侧前扣带回/额内回 & $32 / 10$ & 170 & 6.535 & 0 & 39 & -3 \\
\hline 双侧尾状核/丘脑 & & 737 & 8.762 & -15 & -24 & 9 \\
\hline 双侧后扣带回 & $23 / 31$ & 153 & 5.301 & -3 & -33 & 30 \\
\hline
\end{tabular}

a) $x, y, z$ 为 MNI坐标

表 2 表征主观价值脑区与两种调控方式定向的相关 ${ }^{a)}$

Table 2 Correlations between regions that represented the subjective value and regulatory mode orientations

\begin{tabular}{ccccc}
\hline & \multicolumn{4}{c}{ 表征主观价值的脑区 } \\
\cline { 2 - 5 } & 内侧前额叶 & 腹侧纹状体 & 丘脑 & 后扣带皮层 \\
\hline “评估”定向 & $0.322^{*}$ & -0.015 & -0.143 & 0.089 \\
“行动”定向 & $-0.294^{*}$ & -0.119 & -0.122 & -0.225 \\
\hline
\end{tabular}

a) 数字表示相关系数; * 表示显著性水平 $P<0.0125$ (Bonferroni校正, $0.05 / 4$ 为显著性水平)
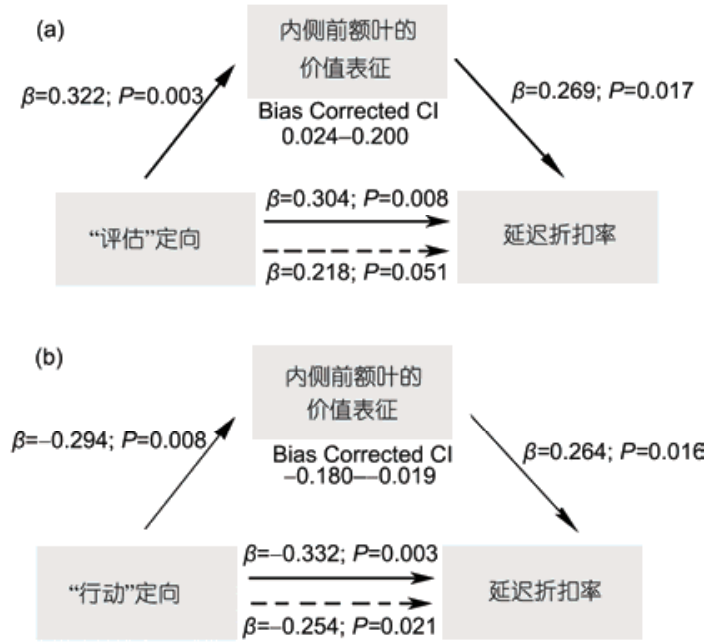

图 4 中介分析结果. (a) 为评估定向对跨期选择影响的中介模型; (b) 为行动定向对跨期选择影响的中介模型

Figure 4 Mediation results. (a) The link between assessment mode and delay discounting was mediated by $\mathrm{mPFC}$ which scaled the subjective value; (b) the link between locomotion mode and delay discounting was also mediated by activity of mPFC
“评估”定向对跨期选择的影响中，表征主观价值的 内侧前额叶的间接效应显著 (bootstrap $=5000$, point estimate $=0.086,95 \%$ percentile bias corrected $\mathrm{CI}$ 为 0.024 0.200; 如图4(a)). 同样地，在“行动”定向对跨 期选择的影响中，表征主观价值的内侧前额叶也存 在显著的间接效应 (bootstrap $=5000$, point estimate $=$ $0.078,95 \%$ percentile bias corrected $\mathrm{CI}$ 为 -0.180 -0.019 ; 如图4(b)). 中介分析的结果表明, 调控方式 对跨期选择的影响可能是通过表征主观价值的内侧 前额叶的活动实现的.

为验证结果的有效性和可靠性，采用分半的方 法(前半被试用于定位分析，后半部分被试用于ROI 分析)进行验证性分析．在定位分析中，同样发现内 侧前额叶，腹侧纹状体，丘脑和后扣带皮层脑区显著 的表征延迟奖赏的主观价值 $(P<0.01 ; \mathrm{FDR}$ 校正; 见图 5及表3). 在ROI分析中, 将表征主观价值的内侧前 额叶作为 ROI, 将其作为中介变量, 进行中介分析. 


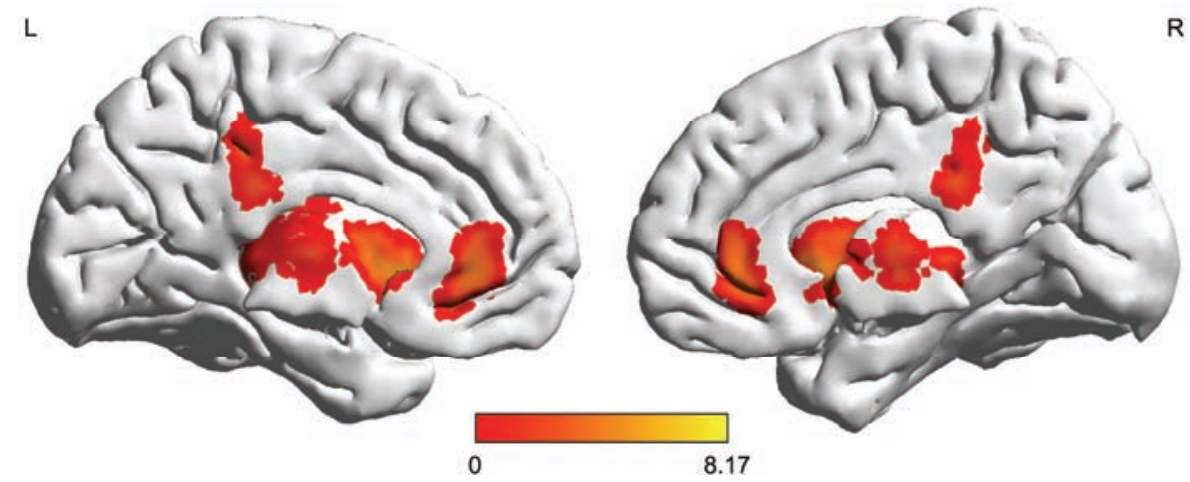

图 5 验证性分析中表征延迟奖赏主观价值的激活脑区 $(P<0.01$, FDR全脑矫正, cluster size $>10)$

Figure 5 Brain regions that represented the subjective value of delayed reward in confirmatory analysis $(P<0.05$, FEW corrected, cluster size $>10)$

表 3 验证性分析中主观价值表征的激活脑区 ${ }^{a}$

Table 3 Activated region that represented the subjective value in confirmatory analysis

\begin{tabular}{lcccccc}
\hline \multicolumn{1}{c}{ 脑区 } & 布鲁德曼分区 & 体素个数 & 激活最强点 $T$ 值 & $x$ & $y$ & $z$ \\
\hline 双侧前扣带回/额内回 & $32 / 24$ & 138 & 4.368 & 9 & 33 & -9 \\
双侧尾状核/丘脑 & & 868 & 8.168 & -9 & 12 \\
双侧后扣带回/楔前叶 & $23 / 31$ & 119 & 3.524 & -6 & -33 & 33 \\
\hline
\end{tabular}

a) $x, y, z$ 为 $\mathrm{MNI}$ 坐标

中介分析同样发现：在“评估”定向对跨期选择的影 响中, 表征主观价值的内侧前额叶的间接效应显著 (bootstrap $=5000$, point estimate $=0.086$, 95\% percentile bias corrected CI为0.016 0.268); 在 “行动”定向对跨期 选择的影响中, 表征主观价值的内侧前额叶也存在显 著的间接效应 (bootstrap $=5000$, point estimate $=0.078$, $95 \%$ percentile bias corrected CI为 $-0.326 \sim 0.011$ ). 验 证性分析的结果表明表征主观价值的内侧前额叶的 活动可能是调控方式影响跨期选择的神经基础.

\section{3 讨论}

本研究使用功能性磁共振成像技术来考察调控 方式影响跨期选择的神经基础. 在行为上发现, “评 估”定向者比“行动”定向者表现出更小的延迟折扣 率; 被试的“评估”定向得分和AUC成正相关, 而“行 动” 定向得分和AUC成负相关. 行为结果和先前的研 究相一致, 表明个体在长久的生活中所形成的调控 方式定向能够预测其延迟折扣率的大小. 脑成像结 果发现, 表征主观价值的内侧前额叶的活动与 “评 估”定向得分成显著的正相关, 而与 “行动”定向得分 成显著的负相关. 更进一步的中介分析表明, 内侧前 额叶的主观价值表征在调控方式与跨期选择之间存 在显著的部分中介效应. 这些结果说明, 调控方式对 跨期选择的影响可能是通过改变延迟奖赏的主观价
值来实现的，而表征主观价值的内侧前额叶则是其 神经基础.

行为结果发现“评估”得分与AUC成正相关，而 “行动”得分和AUC成负相关, “评估”定向者比“行动” 定向者表现出更小的延迟折扣率，这与前人的发现 相一致. Mannetti等人 ${ }^{[10]}$ 通过诱导被试产生特定的 调控方式定向，发现当“评估”定向高于“行动”定向时 被试更多的选择具有长远价值的选项，而当“行动” 定向强于“评估”定向时被试更多的选择“短视”的选 项. 而新近一项研究使用调控方式问卷测量了被试 特质性的调控方式定向，结果同样发现“评估”定向 越强者, 延迟折扣率越小, 而“行动”定向越强者, 延 迟折扣率越大 ${ }^{[11]}$. 这些研究都表明“评估”定向者会 比“行动”定向者表现出更小的延迟折扣率，这可能 是由于他们对延迟奖赏的价值表征不同所造成的.

本研究的脑成像结果发现, 延迟奖赏主观价值 表征的脑区位于内侧前额叶、腹侧纹状体、后扣带回 等脑区. 先前的研究表明这些脑区以双曲线的形式 编码延迟奖赏的主观价值, 它们的激活量随着延迟 奖赏数量的增加而增长, 而随着延迟时间的增加而 下降 ${ }^{[19,38]}$. 个体通过比较主观价值的强弱来决定选 择立即奖赏还是延迟奖赏的. 此外, 虽然内侧前额 叶、腹侧纹状体、丘脑和后扣带皮层这些脑区在跨期 选择中起着表征主观价值的作用，但是有研究表明 
它们可能是表征不同属性的主观价值. 不同领域(包 括跨期选择、风险决策、模糊决策等)的研究共同表 明, 内侧前额叶是整合选项的主观价值神经中 枢 ${ }^{[19,39 \sim 41]}$. 而腹侧纹状体和丘脑作为皮层下重要的 核团, 它们在跨期决策中可能只是加工刺激的奖赏 属性, 这 2 个脑区的活动与冲动性存在紧密的联 系 ${ }^{[27,42,43]}$. 虽然后扣带皮层在跨期决策中的作用不清 楚, 但是有些研究表明该脑区可能跟自传体记忆有 关 ${ }^{[44,45]}$. 因此, 后扣带皮层可能表征的是跟自我相关 的价值属性. 同时, 本研究发现, 表征主观价值的内 侧前额叶的活动与 “评估”得分呈正相关, 而与 “行动” 得分呈负相关. 先前的研究表明, 高“评估”定向者在 做出决策时, 会权衡选项各种属性的价值, 进而做出 最优的决策; 而高 “行动” 定向者只是关注行动的价 值, 缺少对不同价值的权衡 ${ }^{[12,18]}$. 这些发现是跟内侧 前额叶的价值表征的中枢作用相一致的.

本研究中介分析结果发现, 表征主观价值的内 侧前额叶在调控方式与跨期选择之间存在显著的部 分中介作用. 内侧前额叶是跨期选择中价值表征网 络的核心脑区 ${ }^{[19,20]}$. 而内侧前额叶损伤的研究发现, 受损个体在跨期选择中比正常人表现出更大的延迟 折扣率, 这表明该脑区受损会影响到了对延迟奖赏 主观价值的表征, 从而做出更多的冲动选择 ${ }^{[46]}$. 此 外, 跨领域的研究表明内侧前额叶在决策过程中的 作用主要表现在计算各种奖赏或刺激的主观价值, 整合收益与损失 ${ }^{[47 ~ 53]}$. 另一方面, 先前的研究已经 表明, 两种不同的调控方式在价值表征上存在差异. 当面临选择时, “评估”定向者会优先关注选项的价值 而不是选项的可得性, 而”行动” 定向者却会优先关 注选项可得性而不是价值 ${ }^{[12,14]}$. 这表明“评估”定向 者在决策时更加看重事物的价值, 这也符合他们“追 求价值最大化”的原则, 即“do the best”; 而“行动”定 向者更加看重怎样才能参与到行动中去, 即“Just do it”. 因此, “评估”定向者可能比”行动”定向者更擅长 价值加工, 对选项的价值更加敏感, 特别是延迟价值 的表征能力更强. 中介分析的结果说明, “评估”定向 者和”行动”定向者在跨期选择上的差异可能是由于 表征主观价值的内侧前额叶活动上的差异. 也就是 说, “评估”定向者对延迟奖赏的主观价值表征更强, 所以更倾向于选择延迟大奖赏; 而”行动”定向者对 延迟奖赏的主观价值表征较弱, 因而更多选择及时 小奖赏. 因此, 表征主观价值的内侧前额叶可能是调
控方式影响跨期选择的神经基础.

另外, 除了价值表征的加工过程外, 跨期决策还 涉及到认知控制的加工. Figner等人 ${ }^{[54]}$ 使用经㐿磁刺 激(Transcranial Magnetic Stimulation, TMS)技术发现, 当左侧背外侧前额叶 (dorsolateral prefrontal cortex, DLPFC)的功能暂时抑制时, 被试会更多地选择立即 奖赏，但却不影响被试对奖赏价值的评估. 该研究表 明背外侧前额叶在跨期选择中发挥了认知控制的作 用. 另有一些研究表明背外侧前额叶与内侧前额叶 之间的功能耦合可能是跨期选择认知控制加工的神 经基础 ${ }^{[39,49,55]}$. 也有研究者认为背外侧前额叶参与了 跨期选择中决策冲突的过程, 只有在面临选择困难 (延迟奖赏数量在无差别点附近)时才发挥认知调控 的作用 ${ }^{[26,56]}$. 还有研究表明跨期选择涉及到评估和 选择两个阶段, 背外侧前额叶只在选择阶段发挥认 知控制的作用 ${ }^{[57,58]}$. 综合来看, 虽然这些研究都表明 背外侧前额叶参与了跨期决策中认知控制的加工过 程, 但是背外侧前额叶是如何发挥认知控制作用的 仍然存在很大的争议. 本研究只探讨了价值表征的 加工在调控方式与跨期选择之间的作用, 并没有涉 及认知控制的加工. 因此, 将来的研究者可以进一步 探讨评估者和行动者在跨期决策中认知控制的加工 上是否存在差异.

总之, 本研究使用fMRI技术发现, 调控方式对 跨期选择的影响可能是通过改变延迟奖赏的主观价 值来实现的，而表征主观价值的内侧前额叶则是其 神经基础. 本研究将人格特质中的调控方式和跨期 选择联系起来, 并提出了一种调控方式影响跨期决 策的神经解释, 这对于从特质性角度理解跨期选择 的认知神经机制具有重要的科学价值. 同时, 本研究 对于延迟折扣的可塑性也具有重要的启示作用, 具 有相当重要的实践意义. 有研究表明可以通过行为 诱导个体产生特定的调控方式定向 ${ }^{[17,59]}$. 比如, 有研 究者让被试想像个体所经历的典型的高“评估”定向 的行为或高“行动”定向的行为, 发现可以通过这种 方式诱导被试产生高“评估”定向或高“行动”定向. 由 于高“评估”定向者在做出决策时会“三思而后行”, 对 延迟奖赏的价值表征更强，而高“行动”定向者因缺 乏衡量各选项的优劣而会做出一些冲动的选择. 因 此, 将来的研究者可以通过训练个体产生较高的“评 估”定向，增强对延迟奖赏的价值表征，从而使其更 加“目光远大”. 


\section{参考文献}

1 Frederick S, Loewenstein G, O'donoghue T. Time discounting and time preference: A critical review. J Econ Lit, 2002, 40: 351-401

2 Yi R, Gatchalian K M, Bickel W K. Discounting of past outcomes. Exp Clin Psychopharmacol, 2006, 14: 311-317

3 Baumeister R F, Heatherton T F. Self-regulation failure: An overview. Psychol Inq, 1996, 7: 1-15

4 Kim B K, Zauberman G. Perception of anticipatory time in temporal discounting. J Neurosci Psychol Econ, 2009, 2: 91-101

5 Shamosh N A, Gray J R. Delay discounting and intelligence: A meta-analysis. Intell, 2008, 36: 289-305

6 Peters J, Büchel C. Episodic future thinking reduces reward delay discounting through an enhancement of prefrontal-mediotemporal interactions. Neuron, 2010, 66: 138-148

7 Kirby K N. One-year temporal stability of delay-discount rates. Psychon Bull Rev, 2009, 16: 457-462

8 Ohmura Y, Takahashi T, Kitamura N, et al. Three-month stability of delay and probability discounting measures. Exp Clin Psychopharmacol, 2006, 14: 318

9 Anokhin A P, Goloosheykin S, Grant J D, et al. Heritability of delay discounting in adolescence: A longitudinal twin study. Behav Genet, 2011, 41: 175-183

10 Mannetti L, Leder S, Insalata L, et al. Priming the ant or the grasshopper in people's mind: How regulatory mode affects inter-temporal choices. Eur J Soc Psychol, 2009, 39: 1120-1125

11 Mischel W, Shoda Y, Rodriguez M L. Delay of gratification in children. Science, 1989, 244: 933-938

12 Kruglanski A W, Tompson E P, Higgins E T, et al. To "do the right thing" or to "just do it": Locomotion and assessment as distinct selfregulatory imperatives. J Pers Soc Psychol, 2000, 79: 793

13 Higgins E T, Kruglanski A W, Pierro A. Regulatory mode: Locomotion and assessment as distinct orientations. Adv Exp Soc Psychol, 2003, 35: 293-344

14 Kruglanski A W, Orehek E, Higgins E T, et al. Assessment and Locomotion as Independent Determinants in Goal Pursuit. Handbook of Personality and Self-regulation. London: Wiley-Blackwell Press, 2009. 375-402

15 Florack A, Keller J, Palcu J. Regulatory focus in economic contexts. J Econ Psychol, 2013, 38: 127-137

16 Panno A, Pierro A, Lauriola M. Self-regulation predicts risk-taking through people's time horizon. Int J Psychol, 2014, 49: 211-215

17 Pierro A, Leder S, Mannetti L, et al. Regulatory mode effects on counterfactual thinking and regret. J Exp Soc Psychol, 2008, 44: 321-329

18 Avnet T, Higgins E T. Locomotion, assessment, and regulatory fit: Value transfer from "how" to "what". J Exp Soc Psychol, 2003, 39: $525-530$

19 Kable J W, Glimcher P W. The neural correlates of subjective value during intertemporal choice. Nat Neurosci, 2007, 10: 1625-1633

20 Kable J W, Glimcher P W. An "as soon as possible" effect in human intertemporal decision making: Behavioral evidence and neural mechanisms. J Neurophysiol, 2010, 103: 2513-2531

21 Ballard K, Knutson B. Dissociable neural representations of future reward magnitude and delay during temporal discounting. NeuroImage, 2009, 45: 143-150

22 Luo S, Ainslie G, Pollini D, et al. Moderators of the association between brain activation and farsighted choice. NeuroImage, 2012. 59: 1469-1477

23 Sripada C S, Gonzalez R, Phan K L, et al. The neural correlates of intertemporal decision-making: Contributions of subjective value, stimulus type, and trait impulsivity. Hum Brain Mapp, 2011, 32: 1637-1648

24 Yue L Y, Feng T Y, Li S S, et al. The differences of individuals having different regulatory modes on counterfactual thinking: Evidence from an ERP study (in Chinese). Acta Psychol Sin, 2011. 43: 274-282 [岳玲云, 冯廷勇, 李森森, 等. 不同调控方式个体反事实思维 上的差异: 来自 ERP 的证据. 心理学报, 2011, 43: 274-282]

25 Bornovalova M A, Fishman S, Strong D R, et al. Borderline personality disorder in the context of self-regulation: Understanding symptoms and hallmark features as deficits in locomotion and assessment. Pers Individ Dif, 2008, 44: 22-31

26 McClure S M, Laibson D I, Loewenstein G, et al. Separate neural systems value immediate and delayed monetary rewards. Science, 2004, 306: 503-507

27 Jimura K, Chushak M S, Braver T S. Impulsivity and self-control during intertemporal decision making linked to the neural dynamics of reward value representation. J Neurosci, 2013, 33: 344-357

28 Myerson J, Green L, Warusawitharana M. Area under the curve as a measure of discounting. J Exp Anal Behav, 2001, 76: 235-243

29 Shamosh N A, DeYoung C G, Green A E, et al. Individual differences in delay discounting relation to intelligence, working memory, and anterior prefrontal cortex. Psychol Sci, 2008, 19: 904-911

30 Yan C G, Zang Y F. DPARSF: a MATLAB toolbox for "pipeline" data analysis of resting-state fMRI. Front Syst Neurosci, $2010,4: 13$ 
31 Song X W, Dong Z Y, Long X Y, et al. REST: A toolkit for resting-state functional magnetic resonance imaging data processing. PLoS One, 2011, 6: e25031

32 Hayes A F. Introduction to Mediation, Moderation, and Conditional Process Analysis: A Regression-based Approach. New York: Guilford Publications, 2013 Hayes A F. Beyond Baron and Kenny: Statistical mediation analysis in the new millennium. Commun Monogr, 2009, 76: 408-420

Preacher K J, Hayes A F. Asymptotic and resampling strategies for assessing and comparing indirect effects in multiple mediator models. Behav Res Methods, 2008, 40: 879-891

35 Vul E, Harris C, Winkielman P, et al. Puzzlingly high correlations in fMRI studies of emotion, personality, and social cognition. Perspect Psychol Sci, 2009, 4: 274-290

36 Kriegeskorte N, Simmons W K, Bellgowan P S, et al. Circular analysis in systems neuroscience: the dangers of double dipping. Nat Neurosci, 2009, 12: 535-540

37 Bartra O, McGuire J T, Kable J W. The valuation system: A coordinate-based meta-analysis of BOLD fMRI experiments examining neural correlates of subjective value. NeuroImage, 2013, 76: 412-427 Peters J, Büchel C. The neural mechanisms of inter-temporal decision-making: Understanding variability. Trends Cogn Sci, 2011, 15: 227-239 Hare T A, Hakimi S, Rangel A. Activity in dlPFC and its effective connectivity to vmPFC are associated with temporal discounting. Front Neurosci, 2014, 8: 50

40 Pine A, Seymour B, Roiser J P, et al. Encoding of marginal utility across time in the human brain. J Neurosci, 2009, 29: 9575-9581

41 Botvinick M M. Conflict monitoring and decision making: Reconciling two perspectives on anterior cingulate function. Cogn Affect Behav Neurosci, 2007, 7: 356-366

42 Kirby K N, Petry N M. Heroin and cocaine abusers have higher discount rates for delayed rewards than alcoholics or non-drug-using controls. Addiction, 2004, 99: 461-471

43 Hoffman W F, Moore M, Templin R, et al. Neuropsychological function and delay discounting in methamphetamine-dependent individuals. Psychopharmacology, 2006, 188: 162-170

44 Petry N M. Delay discounting of money and alcohol in actively using alcoholics, currently abstinent alcoholics, and controls. Psychopharmacology, 2001, 154: 243-250 Monterosso J, Ainslie G. Beyond discounting: Possible experimental models of impulse control. Psychopharmacology, 1999, 146: 339-347 Sellitto M, Ciaramelli E, Di P G. Myopic discounting of future rewards after medial orbitofrontal damage in humans. J Neurosci, 2010, 30: 16429-16436

47 Basten U, Biele G, Heekeren H R, et al. How the brain integrates costs and benefits during decision making. Proc Natl Acad Sci USA, 2010, 107: 21767-21772

48 Clithero J A, Smith D V, Carter R M, et al. Within-and cross-participant classifiers reveal different neural coding of information. NeuroImage, 2011, 56: 699-708

49 Hare T A, Camerer C F, Rangel A. Self-control in decision-making involves modulation of the vmPFC valuation system. Science, 2009, 324: 646-648

50 Park S Q, Kahnt T, Rieskamp J, et al. Neurobiology of value integration: When value impacts valuation. J Neurosci, 2011, 31: 9307-9314

51 Boorman E D, Behrens T E, Woolrich M W, et al. How green is the grass on the other side? Frontopolar cortex and the evidence in favor of alternative courses of action. Neuron, 2009, 62: 733-743

52 Tom S M, Fox C R, Trepel C, et al. The neural basis of loss aversion in decision-making under risk. Science, 2007, 315: 515-518

53 Wang Q, Luo S, Monterosso J, et al. Distributed value representation in the medial prefrontal cortex during intertemporal choices. J Neurosci, 2014, 34: 7522-7530

54 Figner B, Knoch D, Johnson E J, et al. Lateral prefrontal cortex and self-control in intertemporal choice. Nat Neurosci, 2010, 13: 538-539

55 Steinbeis N, Haushofer J, Fehr E, et al. Development of behavioral control and associated vmPFC-DLPFC connectivity explains children's increased resistance to temptation in intertemporal choice. Cereb Cortex, 2014, 26: 32-42

56 Li N, Ma N, Liu Y, et al. Resting-state functional connectivity predicts impulsivity in economic decision-making. J Neurosci, 2013, 33: 4886-4895

57 Liu L, Feng T, Wang J, et al. The neural dissociation of subjective valuation from choice processes in intertemporal choice. Behav Brain Res, 2012, 231: 40-47

58 Luo S, Ainslie G, Giragosian L, et al. Behavioral and neural evidence of incentive bias for immediate rewards relative to preferencematched delayed rewards. J Neurosci, 2009, 29: 14820-14827

59 Panno A, Lauriola M, Pierro A. Regulatory mode and risk-taking: The mediating role of anticipated regret. PLoS One, 2015, 10: e0143147 


\title{
The neural basis of the effect of regulatory mode on intertemporal choice: the mediating role of $\mathrm{mPFC}$
}

\author{
GUO YiQun ${ }^{1} \&$ FENG TingYong ${ }^{1,2}$ \\ ${ }^{1}$ Research Center of Psychology and Social Development, Faculty of Psychology, Southwest University, Chongqing 400715, China; \\ ${ }^{2}$ Key Laboratory of Cognition and Personality of Ministry of Education, Southwest University, Chongqing 400715, China
}

Decisions that involve tradeoffs between outcomes that occur at different points in time are called intertemporal choice. A consistent finding regarding intertemporal choice is that delayed outcomes are discounted relative to immediate ones. The extent of preference for delayed rewards is captured by delay discounting rate, which expresses that the subjective value of a delayed reward declines as a function of delay. It is generally thought that people are fairly stable in their intertemporal preference, and it has been suggested that they may have a genetic component. Previous studies have shown that the chronic regulatory mode orientation can affect intertemporal choice. Steeper delay discounting is related to lower assessment orientation, while related to higher locomotion orientation. However, the cognitive and neural mechanism of the effect of regulatory mode on intertemporal choice is still unclear. Recent neuroimaging studies have identified a valuation network (such as ventral striatum, posterior cingulate cortex, and medial prefrontal cortex) in delay discounting, which is the neural computation and representation of the subjective values of rewards. Thus, we investigated the role of the valuation network in the relation between regulatory mode and delay discounting.

In the present study, we used functional magnetic resonance imaging (fMRI) to investigate the neural basis of this effect. We used two different approaches to examine the link between regulatory mode and delay discounting. First, we split participants into two groups of assessors and locomotors according to relative strength of regulatory orientations, and compared the difference between the two groups. We found that locomotors showed a steeper delay discounting than assessors. Our second approach examined this effect in a continuous rather than a categorical manner. Correlation results showed that delay discounting was negatively correlated with assessment scores, but positively correlated with locomotion scores. These behavioral findings suggest that individual differences in regulatory mode could predict delay discounting, and are consistent with previous studies.

Our neuroimaging results first indicated that neural activity in medial prefrontal cortex (mPFC), ventral striatum (VS), thalamus, and posterior cingulate cortex (PCC) tracks the subjective value of delayed rewards. We next computed the correlations between neural activity in these regions and regulatory mode. Correlation results showed that only the activity in $\mathrm{mPFC}$ which scales the subjective value was positively correlated with assessment scores, but negatively correlated with locomotion scores. Furthermore, mediation analysis showed that neural activity in mPFC partially mediated the link between regulatory mode and delay discounting. These results suggested that regulatory mode is associated with intertemporal choice in part because of processes instantiated in $\mathrm{mPFC}$, a region known to represent the subjective value.

In short, we found that the effect of two distinct regulatory modes on delay discounting have different patterns, and were mediated by neural activity in $\mathrm{mPFC}$ which represents subjective value of delayed reward. The present study indicated that $\mathrm{mPFC}$ is a neural locus for determining individual differences in delay discounting, and provides neural basis for explaining the association between regulatory mode and delay discounting.

intertemporal choice, regulatory mode, assessment mode, locomotion mode, medial prefrontal cortex (mPFC), functional magnetic resonance imaging (fMRI)

doi: 10.1360/N972016-00361 